\title{
Cross-Sectional Risk Factors of Anterior Knee Pain in Adolescents
}

\author{
Gregory Borschneck ${ }^{1}$, Laura St. John ${ }^{2}$, Kristy Brundage ${ }^{3}$ and Daniel Patrick Borschneck ${ }^{4 *}$ \\ ${ }^{1}$ Department of Biomedical and Molecular Sciences, Faculty of Health Sciences, School of Medicine, Queen's University, \\ Kingston, ON, Canada, ${ }^{2}$ Infant and Child Health Lab, Faculty of Kinesiology and Physical Education, University of Toronto, \\ Toronto, ON, Canada, ${ }^{3}$ Faculty of Health Sciences, School of Rehabilitation Therapy, Queen's University, Kingston, ON, \\ Canada, ${ }^{4}$ Department of Surgery, Faculty of Health Sciences, School of Medicine, Queen's University, Kingston, ON, Canada
}

\section{OPEN ACCESS}

Edited by:

Tonia C. Onyeka,

University of Nigeria, Nigeria

Reviewed by:

Francisco Reinoso-Barbero, University Hospital La Paz, Spain

Poh-Heng Chong,

HCA Hospice Care, Singapore

*Correspondence:

Daniel Patrick Borschneck dan.borschneck@kingstonhsc.ca

Specialty section:

This article was submitted to

Pediatric Pain

a section of the journal

Frontiers in Pain Research

Received: 03 June 2021 Accepted: 22 September 2021

Published: 22 October 2021

Citation:

Borschneck G, St. John L, Brundage $K$ and Borschneck DP (2021) Cross-Sectional Risk Factors of Anterior Knee Pain in Adolescents.

Front. Pain Res. 2:720236

doi: 10.3389/fpain.2021.720236
Background: Anterior knee pain is a common complaint amongst adolescents, which can both be persistent, and in some cases, disabling. This study investigated a series of potential risk factors potentially linked to the onset of anterior knee pain.

Methods: Questionnaires were distributed amongst 367 10-15 years-olds enrolled in the local school board. These surveys included questions on sex, age, sport participation, and history of anterior knee pain verified by a physician. Bivariate correlations and a binomial logistic regression were conducted. Overall rate of AKP in the population studied was $7.4 \%$. The results indicated that past history of knee pain, age, and increased sports participation significantly correlated with increased risk of AKP. AKP was significantly more common in females than males. While sex, height, age, overall sport participation, participation in specific sports, and history of knee injury all contributed to the binomial model.

Keywords: patella-femoral pain syndrome, anterior knee pain (AKP), sport, pediatric pain, joint pain, chronic pain, musculoskeletal pain

\section{INTRODUCTION}

Anterior knee pain (AKP) describes a group of persistent (1) and occasionally disabling conditions afflicting the anterior aspect of either one or both knees (2). AKP is often used interchangeably with the patellofemoral joint pain (PFJP) (3), but also encompasses broader conditions including patellar tendinopathy, Osgood Schlatter's disease, and pain of the anterior tibiofemoral joint. AKP is relatively uncommon in children $(<10$ years old $)(4,5)$; however, prevalence significantly increases throughout adolescence impacting $20-30 \%$ of this population $(5,6)$. Adolescents often report this pain as mild; however, in some cases (13\%) it may be a significant disability (5) associated with early-onset patellofemoral osteoarthritis (7). AKP is responsible for between 10 and $40 \%$ of all physiotherapy visits made by adolescents $(8,9)$ and often results in maladaptive behaviors including withdrawal from sports (10). Despite this prevalence and severity, the majority of research on AKP takes place in adults rather than adolescents (11).

AKP has been linked with poor biomechanical alignment across the trunk, hip, knee, and ankle joints (12). Numerous intrinsic factors are thought to relate with poor alignment, including lack of flexibility (13), poor muscular strength $(14,15)$, natural alignment $(15,16)$, and poor motor control $(12,17)$; such patterns and high-risk malalignment is known to be particularly common amongst females (12). While intrinsic risk factors surrounding alignment have received considerable focus in recent meta-analyses $(18,19)$, it is also important to consider how extrinsic risk factors related to loading quantity relate to AKP rates. Amongst adolescents, organized sporting participation is 
known to increase (20); those involved in sports throughout adolescence demonstrate increased levels of overall, moderate, and vigorous physical activity in both sexes (less prominent in girls) (21). Despite the link between overall loading and AKP risk, past cross-sectional studies have not demonstrated a consistent link between sports participation status and AKP development in adolescents $(5,6,22)$. These studies did not consider type of sport, only overall sporting behavior; as some sports have a known association with $\operatorname{AKP}(23,24)$ it is possible that cultural differences in prevalence of specific sports has impacted this relationship.

Accordingly, the purpose of this study was to determine if overall sporting participation or involvement in specific sports place adolescents at an increased risk of AKP.

\section{METHODS}

\section{Population}

A cross-sectional survey was performed between May 2001 to June 2001. Participants were a convenience sample of pre-adolescent and adolescent youth (10-15 years) enrolled in three public primary schools and two public secondary schools throughout Kingston, Ontario, Canada. Following the permission of the schools, students in randomly selected physical education classes were approached in the presence of their teachers; these students were handed a brochure explaining the study, a parental consent form, and data questionnaire (Figure 1). The population of students sampled

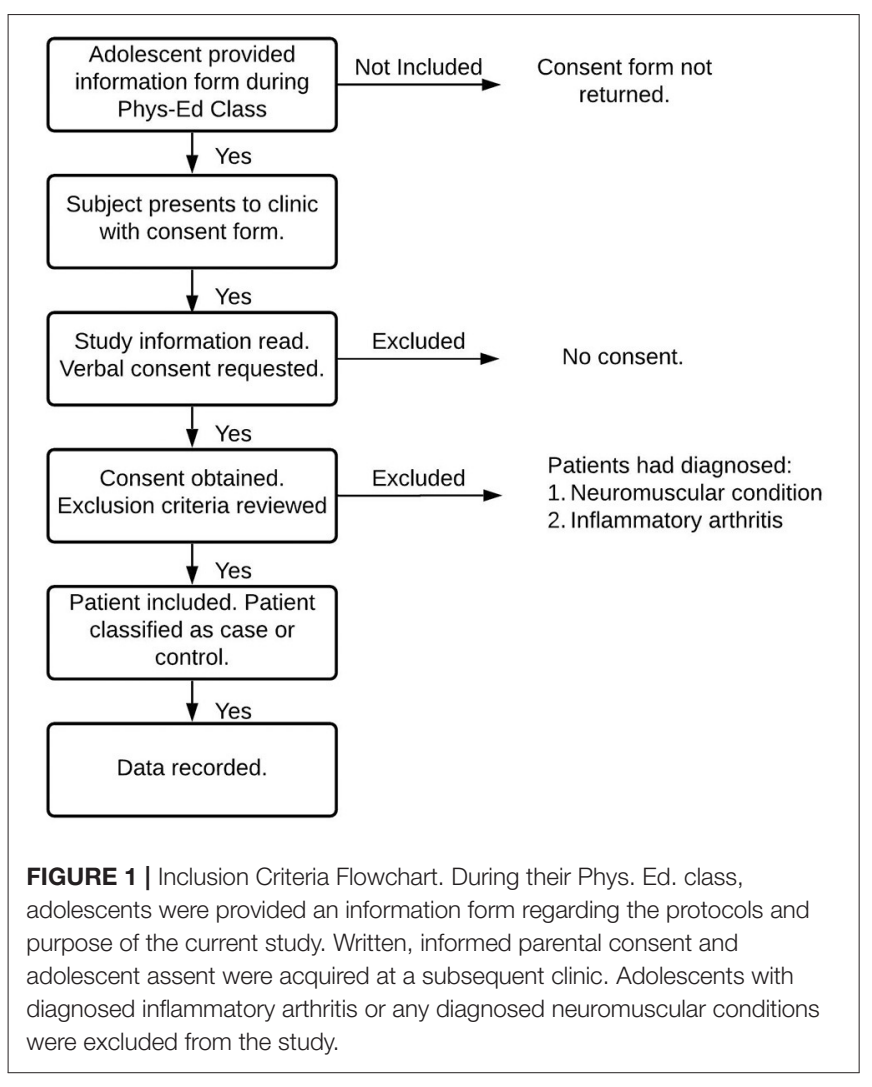

excluded those who were not enrolled in general classes due to special needs requirements, were diagnosed with neuromuscular conditions, or inflammatory arthritis; study designers were blind to any prevalence of AKP during selection. Prior to data acquisition, ethics approval was obtained from both the Queens University and Affiliated Teaching Hospitals Health Sciences Research Ethics Board and the Limestone District School Board. Participants and parents were informed about the purpose and methodology of the study; informed written consent was obtained from the parent and informed verbal assent from the child before enrollment. Only students who verbally assented to inclusion and returned both their completed surveys and parent consent forms were considered for participation in this study.

\section{Metrics}

Demographic information including age, height, and sex were recorded. History of medically diagnosed knee injury and the dichotomous presence/absence of knee pain localized to the anterior knee which was significant enough to limit activity for more than one week over the past year were also documented. Participants were asked via an open-ended text box to list the sporting activities (outside of physical education class) that they spent a significant amount of time participating in over the past year; it was hoped that allowing a participant to subjectively determine the number of sports that they participated in would allow them to include any unorganized or "pick-up" sports they played. An additional combinate categorical variable termed "Total Number of Sports" (reflecting the total number of sports the adolescent played) was also analyzed; due to the limited number of athletes playing four (11), five (7), or six (3) sports, these subjects have been grouped into a single category as $\geq 3$.

\section{Procedure}

Students completed a self-report questionnaire, which was returned to the research team. Upon return of the self-report questionnaire, a physician (who was a research team member) was available to aid in question comprehension, ensure accurate responses, and gather missing data. Presence of AKP, based on the definition listed in section 2.2 was confirmed during the follow up interview. Height was recorded for each subject following return of survey and consent form.

\section{Statistical Analysis}

All statistical analyses were performed using SPSS 27.0.1.0. Any subjects with missing data were excluded from the analysis. Dichotomous independent variables sex, participation in any sports and binary participation in specific sports were analyzed along with the binary dependent variable of knee pain in an exact Fisher's Test. An exact Fisher's test was chosen due to concerns that some groups would involve fewer than five participants. Along with the significance of the exact Fisher's test, the phi coefficient was recorded as a measure of association. The ordinal variable total sport involvement was analyzed against knee pain using a Pearson's Chi-Square analysis due to the non-binary nature of this data. Continuous independent variables height and age were tested for association against the independent variable 
TABLE 1 | Demographics.

\begin{tabular}{lc}
\hline Descriptor & $\boldsymbol{N}$ \\
\hline Total Subjects & 367 \\
Sex & \\
Females & 207 \\
$\quad$ Males & 160 \\
Age group (years) & \\
10 & 11 \\
11 & 44 \\
12 & 52 \\
13 & 57 \\
14 & 149 \\
15 & 55 \\
Current number of extra-curricular sports played & \\
0 & 280 \\
1 & 28 \\
2 & 38 \\
3 & 17 \\
$\geq 3$ & 21 \\
Anterior Knee Pain & \\
Yes & 27 \\
No & 340 \\
Knee injury & \\
No & 18 \\
\hline
\end{tabular}

Overall, 367 participants were included in this study, There were more females $(n=$ 207) than males $(n=160)$ in this study's sample. The greatest number of participants were 14 years old $(n=149)$, while the fewest were 10 years old $(n=11)$. The majority of participants in this study did not play sports $(n=280)$. AKP was reported in 27 participants.

of knee pain using a Spearman's rho correlation; significance and correlation coefficient.

A binomial logistic regression was conducted due to the binary nature of the dependent variable. All variables recorded in the study were initially included and remained in the model if they contributed according to the results of the wald test. The binomial logistic regression method was chosen because multiple independent variables were considered, and due to the dichotomous nature of the dependent variables measured. A correlation between predictor variables was run alongside the regression to confirm that the assumption of independence was upheld; in this correlation the combined metric "total sport involvement" was found to moderately correlate with several other factors and so was removed from the hierarchical regression.

\section{RESULTS}

The final study sample included 367 participants (see Table 1).

\section{Demographics}

There were significantly more females $(n=207)$ than males $(n=160)$ enrolled; the mean age of the sample was 13.2
TABLE 2 | Incidence per Group.

\begin{tabular}{|c|c|c|}
\hline Variable & Total count & Percent with AKP \\
\hline $\mathrm{Age}^{\dagger}$ & 367 & $7.36 \%$ \\
\hline 10 & 11 & $0.00 \%$ \\
\hline 11 & 44 & $2.27 \%$ \\
\hline 12 & 52 & $5.77 \%$ \\
\hline 13 & 57 & $3.51 \%$ \\
\hline 14 & 149 & $10.74 \%$ \\
\hline 15 & 55 & $9.09 \%$ \\
\hline $\operatorname{Sex}^{\dagger}$ & 367 & $7.36 \%$ \\
\hline Male & 160 & $3.75 \%$ \\
\hline Female & 207 & $10.14 \%$ \\
\hline Knee Injury & 18 & $61.11 \%$ \\
\hline Total Sport ${ }^{\dagger \dagger}$ & 87 & $22.99 \%$ \\
\hline 0 & 280 & $2.50 \%$ \\
\hline 1 & 28 & $21.43 \%$ \\
\hline 2 & 38 & $15.79 \%$ \\
\hline 3 & 17 & $23.81 \%$ \\
\hline$>3$ & 21 & $38.10 \%$ \\
\hline Sports $^{\dagger \dagger}$ & 87 & $22.99 \%$ \\
\hline Soccer & 29 & $17.24 \%$ \\
\hline Basketball & 31 & $29.03 \%$ \\
\hline Baseball & 17 & $29.41 \%$ \\
\hline Hockey & 14 & $7.14 \%$ \\
\hline Swimming & 27 & $11.11 \%$ \\
\hline Cross-Country & 14 & $28.57 \%$ \\
\hline Riding & 4 & $50.00 \%$ \\
\hline Skating & 7 & $14.29 \%$ \\
\hline Volleyball & 25 & $28.00 \%$ \\
\hline
\end{tabular}

This table depicts incidence of AKP across the different groups in this study along with the total count for the given condition. Highest prevalence was amongst those with past knee injuries. Incidence of knee pain notably increases with age and amongst females. AKP, Anterior Knee Pain.

${ }^{\dagger}$ Rate of AKP reported for entire sample; ${ }^{\dagger \dagger}$ Rate of AKP reported for all engaged in sport.

years $(\mathrm{SD}=1.36)$, with a range from 10 to 15 years. Twentyseven of the participants $(7.4 \%)$ reported knee pain, and $18 / 367$ (4.9\%) reported a knee injury in the previous year. Of the 367 participants, $87(23.7 \%)$ of the participants were involved in extracurricular sports with the most commonly played sports being basketball $(n=31)$, soccer $(n=29)$, and swimming $(n=$ 27). Descriptive characteristics are further explained on Table 1.

Incidence of AKP was greatest amongst 14-year-olds (10.74\%) and non-existent amongst 10 -year-olds (0.00\%). AKP was most prevalent amongst those with a history of knee injury $(61.11 \%)$ and generally increased in those who played any sport (22.99\%). Table 2 displays specific incidence for each group.

\section{Individual Associations}

Statistically significant associations were observed between both AKP and sex $(r=-0.121, p=0.02)$, AKP and age $(r=0.112$, $p=0.033)$, and AKP and total number of sports played $(r=$ $0.329, p<0.001)$. Females $(r=0.121, p=0.02)$ were more likely to experience knee pain than males. A moderate risk for knee 
TABLE 3 | Binary logistic regression results.

\begin{tabular}{|c|c|c|c|c|c|c|}
\hline Variable & B & SE & $P$ & OR & \multicolumn{2}{|c|}{$95 \% \mathrm{Cl}$} \\
\hline Age & 0.372 & 0.284 & 0.190 & 1.451 & 0.832 & 2.531 \\
\hline $\operatorname{Sex}^{\dagger}$ & 0.033 & 0.676 & 0.961 & 1.034 & 0.275 & 3.889 \\
\hline Height & -0.020 & 0.032 & 0.533 & 0.980 & 0.921 & 1.044 \\
\hline 1 Sport & 2.945 & 0.816 & $<0.001^{\star \star}$ & 19.019 & 3.839 & 94.216 \\
\hline 2 Sports & 4.814 & 1.347 & $0.001^{*}$ & 75.585 & 5.393 & 1059.375 \\
\hline$\geq 3$ Sports & 6.134 & 2.059 & $0.008^{*}$ & 165.756 & 2.927 & 9386.299 \\
\hline 4 Sports & 9.665 & 3.213 & $0.006^{*}$ & $9,684.151$ & 17.823 & 5261828.97 \\
\hline Knee Injury & 2.914 & 0.739 & $<0.001^{\star *}$ & 18.422 & 4.327 & 78.438 \\
\hline Hockey & -2.324 & 1.406 & 0.098 & 0.098 & 0.006 & 1.541 \\
\hline Riding & -0.625 & 1.769 & 0.724 & 0.535 & 0.017 & 17.171 \\
\hline Skating & -1.751 & 2.322 & 0.451 & 0.174 & 0.002 & 16.433 \\
\hline Soccer & -4.005 & 1.899 & $0.035^{*}$ & 0.018 & 0.000 & 0.753 \\
\hline Swimming & -3.060 & 1.243 & $0.014^{*}$ & 0.047 & 0.004 & 0.536 \\
\hline Volleyball & -1.448 & 1.227 & 0.238 & 0.235 & 0.021 & 2.605 \\
\hline
\end{tabular}

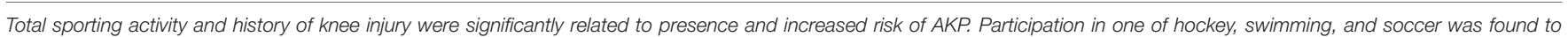
serve a significantly protective effect against AKP. Football and dance demonstrated a Wald statistic of zero and accordingly were not included in this table.

OR, Odds Ratio; Cl, Confidence Interval.

${ }^{\dagger}$ Sex data is relative to Males. ${ }^{*} p<0.05 ;{ }^{* *} p<0.001$.

pain was noted among those involved in extracurricular sports $(r=0.352, p<0.001)$ compared to those not participating in sports. A moderately increased risk for knee pain was observed in participants with a history of knee injury $(r=0.468, p<$ $0.001)$. Height was not found to significantly relate to risk of developing AKP $(p=0.646)$ both overall and after controlling for participant age.

Participation in soccer $(r=0.111, p<0.034)$, basketball $(r=$ $0.252, p<0.001)$, baseball $(r=0.186, p=0.005)$, cross-country $(r$ $=0.162, p=0.014)$, riding $(r=0.171, p=0.029)$, and volleyball ( $r=0.214, p=0.001)$ all were found to slightly but significantly increase risk of developing AKP. Total sports played $(r=0.352$, $p<0.001)$ significantly positively correlated with increased rates of AKP. When sporting involvement was controlled for, participation in soccer, football, hockey, swimming, gymnastics, and skating all correlated slightly negatively with AKP, whereas participation in basketball, baseball, cross-country, riding, and volleyball correlated slightly positively with AKP.

\section{Binomial Logistic Regression}

A binomial logistic regression was performed to predict knee pain based on age, sex, history of knee injury, height, number of sporting activities, and the dichotomous nature of all sports recorded. As the only continuous variables involved in this regression, height and age were tested a priori using the BoxTidwell transformation to confirm that there was no violation of the assumption of the linearity of logit. Football and gymnastics were subsequently removed from this model as they returned Wald statistics of 0 . This binomial logistic regression was a statistically significant improvement $(p=0.00)$ over an unconditional model.

When all variables in this study were accounted for, history of knee injury ( $\mathrm{OR}=18.422, B=2.914)$ and total sporting involvement at all levels (one sport: $\mathrm{OR}=19.019, B=2.945$; two sports: $\mathrm{OR}=75.585, B=4.814$; three sports: $\mathrm{OR}=165.756, B=$ $6.134 ; 4$ sports: $\mathrm{OR}=9,684.151 ; B=9.665)$ remained significant (Table 3). Two sports were significant in the model, participation in swimming $(\mathrm{OR}=0.047, B=-3.060)$ and soccer $(\mathrm{OR}=$ $0.018, \mathrm{~B}=-4.005)$ were both found to demonstrate a protective effect on AKP. All metrics involved in the model are described in Table 3.

\section{DISCUSSION}

The current study sought to identify risk factors associated with and rates of AKP in pre-adolescents and adolescents, representing one of the largest cross-sectional samples to date. AKP's prevalence in this study (7.4\%) was lower than other reports on the general adolescent population. A study by Fairbank described self-reported knee pain at a prevalence of $30 \%$ within a population aged 13-17 years old; this study focused on patellofemoral joint pain but also included other conditions such as Osgood-Schlatter's disease (6). Vahasarja reported the prevalence of a generalized anterior knee pain in 
$18.5 \%$ of their sample aged 14-15 years (5). AKP was most prevalent in our study's 14-year-old population, of which $10 \%$ had AKP; this prevalence is lower than reported by Fairbanks and Vahasarja. As both the Fairbanks and Vahasarja studies were conducted in Europe more than 5 years before this study, perceptions surrounding pain may differ. It is unclear whether these temporal variations and subtle differences in descriptions and categorization of pain impacted reported prevalence.

\section{Age}

This study found a slight but significant positive relationship between age and prevalence of AKP. Prevalence of AKP within the sample was much greater amongst participants 14 and older (10.37\%) compared to those age 13 and younger (3.89\%). Vähäsarja (5) generally agree with such a finding, demonstrating that postpubescent (14-15-year-olds) present with a significantly increased rate of knee pain compared to prepubescent children (9-10 years old). There are many potential explanations for this finding ranging from the onset of puberty to the accumulation of damage.

\section{Sex}

Females' tendency to report an increased prevalence of knee pain is consistent with past observations (25-27). Increased prevalence of AKP in female adolescents not only exists in the general population but also among athletes. Amongst pubertal females, tissue properties are known to associate with biomechanical changes; these changes may associate with the increased incidence of AKP this study found in adolescent females (28).

Alike in the bivariate analysis being a female increased risk of reporting AKP in the linear model; however, this effect was not significant. This may be due to a statistically significant increase in the prevalence of past knee injuries amongst female participants and a statistically significant decrease in hockey involvement, which was one of the sports that demonstrated protective effects against AKP development.

\section{Sport Participation}

Participation in organized sports was moderately associated with knee pain. This finding is not consistent with the findings of Fairbank, who did not identify a sports/knee pain association (6). Fairbank inquired about sports involvement by asking respondents "Do you like to play sport" with the possible responses: "as much as possible," "average," "as little as possible." Forty-five percent of respondents were in the first category "as much as possible," and 50\% played an average amount (6). The structure of the Fairbank study question may have led to misclassification of this sports involvement variable. Many adolescents may wish to play sports "as much as possible" but play little in reality, making comparisons difficult. Generally, studies that focus on elite athletic pediatric populations demonstrate an increased study-wide incidence of AKP compared to these broader studies.

While increasing sport participation was found to increase risk of AKP (increasing OR, see Table 3) a matched-crosssectional study by Rathleff et al. disagrees, finding that those with
AKP withdrew, reducing their sporting participation (29). As the sample Rathleff et al. tested was considerably older (ages 15-19) than those assessed in this study this disagreement may reflect a temporal relationship between sport and AKP. Specifically, the marked increase in AKP incidence once subjects reach 14 years of age (Table 2) may lead to the increased dropout that Rathleff et al. reported between years 15-19 (29).

This study is one of few to examine which sports may increase or decrease risk of AKP within the same population. Research regarding sport-based prevalence of AKP have focused primarily on a select few sports, such as dance. The prevalence of AKP across both the general adolescent population and all sports studied here urges authors to consider how under-researched activities like horseback riding relate to AKP risk and compare to other sports.

\section{Knee Injury}

Knee injury within the past year was found to significantly associate with reported AKP both in bivariate assessments and linear modeling. A major systematic review of all research in this field suggested that several of the biomechanical patterns known to increase risk of AKP are particularly common in injured knees (30). As outlined above rate of knee injury within the past year was found to be significantly greater amongst females. These results concur with past research which suggests that in most sports females are equally or more likely to experience knee injuries -particularly ACL tears- than males (31).

\section{Limitations}

One limitation of this study was its population. As a true cross-sectional study the number of participants with AKP was considerably lower than those unaffected, limiting the generalizability of these findings. The disproportionate nature of the control and AKP groups were to be expected in the population of interest. While this study examined the overall number of sports a participant played, it did not account for the hours and rigor involved in that activity. Accounting for sporting intensity may help explain why risk of AKP changes relatively little after an athlete is already engaged in sport; this would better account for the risk of AKP faced during early specialization (32-34). It is important to note that a small percentage $(31 \%)$ of our population was engaging in extracurricular sport which greatly differs from what has been seen in Canadian Youth (35). While this disparity could impact the generalizability of findings, it does reflect the population that we sampled who tended to be in their young teens when sport drop out occurs more frequently $(36,37)$. Lastly, the data involved in this study was collected in 2001 and any cultural changes surrounding pain or sporting behavior since then may impact generalizability.

Despite these limitations, the results of this study are largely generalizable. The disproportionate number of females in the study population was controlled for during modeling. Further, as all adolescents are required to take physical education at the ages sampled there is no reason to suggest that this study was not open to the general population of adolescents in the grades sampled. 


\section{CONCLUSIONS}

Analysis revealed that the most potent risk factor for AKP pain is past, treated, knee injuries which seemed to predispose females in particular to the development AKP. Athletes who participated in some sports were at heightened risk of developing AKP, although this did not scale with the number of sports played. While non-significant, different sports variably influenced risk of AKP development; future studies should look to compare these sports with a larger sample size to determine how involvement impacts overall risk. Future studies should attempt to assess how pain severity relates to the factors evaluated in this study; understanding how different groups of adolescents respond to different severities of AKP may help develop early warning signs for this condition.

\section{DATA AVAILABILITY STATEMENT}

The original contributions presented in the study are included in the article/Supplementary Material, further inquiries can be directed to the corresponding author/s.

\section{ETHICS STATEMENT}

The studies involving human participants were reviewed and approved by Queens University and Affiliated Teaching

\section{REFERENCES}

1. Kastelein M, Luijsterburg PA, Heintjes EM, van Middelkoop M, Verhaar JA, Koes BW, et al. The 6-year trajectory of non-traumatic knee symptoms (including patellofemoral pain) in adolescents and young adults in general practice: a study of clinical predictors. Br J Sports Med. (2015) 49:4005. doi: 10.1136/bjsports-2014-093557

2. Sisk D, Fredericson M. Update of risk factors, diagnosis, and management of patellofemoral pain. Curr Rev Musculoskelet Med. (2019) 12:53441. doi: 10.1007/s12178-019-09593-z

3. Biber R, Gregory A. Overuse injuries in youth sports: is there such a thing as too much sports? Pediatr Ann. (2010) 39:286-92. doi: 10.3928/00904481-20100422-09

4. Steinberg N, Tenenbaum S, Hershkovitz I, Zeev A, Siev-Ner I. Lower extremity and spine characteristics in young dancers with and without patellofemoral pain. Res Sports Med. (2017) 25:166-80. doi: 10.1080/15438627.2017.1282355

5. Vähäsarja V. Prevalence of chronic knee pain in children and adolescents in northern Finland. Acta Paediatr. (1995) 84:803-5. doi: 10.1111/j.1651-2227.1995.tb13760.x

6. Fairbank JC, Pynsent PB, van Poortvliet JA, Phillips H. Mechanical factors in the incidence of knee pain in adolescents and young adults. J Bone Joint Surg $B r$. (1984) 66:685-93. doi: 10.1302/0301-620X.66B5.6501361

7. Utting MR, Davies G, Newman JH. Is anterior knee pain a predisposing factor to patellofemoral osteoarthritis? Knee. (2005) 12:362-5. doi: 10.1016/j.knee.2004.12.006

8. Stanitski CL. Anterior knee pain syndromes in the adolescent. Instr Course Lect. (1994) 43:211-20.

9. Clark DI, Downing N, Mitchell J, Coulson L, Syzpryt EP, Doherty M. Physiotherapy for anterior knee pain: a randomised controlled trial. Ann Rheum Dis. (2000) 59:700-4. doi: 10.1136/ard.59.9.700
Hospitals Health Sciences Research Ethics Board. Written informed consent to participate in this study was provided by the participants' legal guardian/next of kin.

\section{AUTHOR CONTRIBUTIONS}

GB performed statistical analysis based on data obtained within DB's thesis. GB and LS wrote and edited the manuscript with consult from DB. KB was integral to data collection. DB conducted the original thesis from which this study is derived. All authors contributed to the article and approved the submitted version.

\section{ACKNOWLEDGMENTS}

The authors of this study would like to acknowledge the contributions of Dr. Will Pickett, who supervised the broader thesis (38) that included this project. The authors also wish to thank the Kingston Frontenac Addington and Lennox school board for facilitating this study's access to its sample population.

\section{SUPPLEMENTARY MATERIAL}

The Supplementary Material for this article can be found online at: https://www.frontiersin.org/articles/10.3389/fpain. 2021.720236/full\#supplementary-material

10. Rathleff MS, Rasmussen S, Olesen JL. Unsatisfactory long-term prognosis of conservative treatment of patellofemoral pain syndrome. Ugeskrift for laeger. (2012) 174:1008-13.

11. Rathleff M. Patellofemoral Pain During Adolescence: Much More Prevalent Than Appreciated. Aalborg: BMJ Publishing Group Ltd and British Association of Sport and Exercise Medicine (2016).

12. Nakagawa TH, Moriya É T, Maciel CD, Serrão AF. Frontal plane biomechanics in males and females with and without patellofemoral pain. Med Sci Sports Exerc. (2012) 44:1747-55. doi: 10.1249/MSS.0b013e318256903a

13. Hamstra-Wright KL, Earl-Boehm J, Bolgla L, Emery C, Ferber R. Individuals with patellofemoral pain have less hip flexibility than controls regardless of treatment outcome. Clin J Sport Med. (2017) 27:97103. doi: 10.1097/JSM.0000000000000307

14. Tyler TF, Nicholas SJ, Mullaney MJ, McHugh MP. The role of hip muscle function in the treatment of patellofemoral pain syndrome. Am J Sports Med. (2006) 34:630-6. doi: 10.1177/0363546505281808

15. Lankhorst NE, Bierma-Zeinstra SMA, Van Middelkoop M. Factors associated with patellofemoral pain syndrome: a systematic review. Brit J Sports Med. (2013) 47:193-206. doi: 10.1136/bjsports-2011-090369

16. Emami MJ, Ghahramani MH, Abdinejad F, Namazi H. Q-angle: an invaluable parameter for evaluation of anterior knee pain. Arch Iranian Med. (2007) 10:24-36.

17. Mellor R, Hodges PW. Motor unit synchronization is reduced in anterior knee pain. J Pain. (2005) 6:550-8. doi: 10.1016/j.jpain.2005.03.006

18. Hart HF, Barton CJ, Khan KM, Riel H, Crossley KM. Is body mass index associated with patellofemoral pain and patellofemoral osteoarthritis? A systematic review and meta-regression and analysis. Bri J Sports Med. (2017) 51:781-90. doi: 10.1136/bjsports-2016-096768

19. Neal BS, Lack SD, Lankhorst NE, Raye A, Morrissey D, van Middelkoop M. Risk factors for patellofemoral pain: a systematic review and meta-analysis. Brit J Sports Med. (2019) 53:270. doi: 10.1136/bjsports-2017-098890 
20. Bridge MW, Toms MR. The specialising or sampling debate: a retrospective analysis of adolescent sports participation in the UK. J Sports Sci. (2013) 31:87-96. doi: 10.1080/02640414.2012.721560

21. Telford RM, Telford RD, Cochrane T, Cunningham RB, Olive LS, Davey R. The influence of sport club participation on physical activity, fitness and body fat during childhood and adolescence: the look longitudinal study. J Sci Med Sport. (2016) 19:400-6. doi: 10.1016/j.jsams.2015.04.008

22. Rathleff CR, Baird WN, Olesen JL, Roos EM, Rasmussen S, Rathleff MS. Hip and knee strength is not affected in 12-16 year old adolescents with patellofemoral pain - a cross-sectional population-based study. PLOS ONE. (2013) 8::e79153. doi: 10.1371/journal.pone.0079153

23. Smith PJ, Gerrie BJ, Varner KE, McCulloch PC, Lintner DM, Harris JD. Incidence and prevalence of musculoskeletal injury in ballet: a systematic review. Orthop J Sports Med. (2015) 3:2325967115592621. doi: 10.1177/2325967115592621

24. Nejati P, Forogh B, Moeineddin R, Baradaran HR, Nejati M. Patellofemoral pain syndrome in Iranian female athletes. Acta Med Iranica. (2011) 49:169-72.

25. Fulkerson JP, Arendt EA. The female knee-anterior knee pain. Conn Med. (1999) 63:661-4.

26. Fulkerson JP. Patellofemoral pain disorders: evaluation and management. J Am Acad Orthop Surg. (1994) 2:12432. doi: 10.5435/00124635-199403000-00006

27. Thomee R. A comprehensive treatment approach for patellofemoral pain syndrome in young women. Phys Ther. (1997) 77:1690703. doi: $10.1093 / \mathrm{ptj} / 77.12 .1690$

28. Wild CY, Munro BJ, Steele JR. Higher anterior knee laxity influences the landing biomechanics displayed by pubescent girls. J Sports Sci. (2017) 35:15965. doi: 10.1080/02640414.2016.1159715

29. Rathleff MS, Rathleff CR, Olesen JL, Rasmussen S, Roos EM. Is knee pain during adolescence a self-limiting condition? Prognosis of patellofemoral pain and other types of knee pain. Am J Sports Med. (2016) 44:116571. doi: 10.1177/0363546515622456

30. Weiss K, Whatman C. Biomechanics associated with patellofemoral pain and ACL injuries in sports. Sports Med. (2015) 45:132537. doi: 10.1007/s40279-015-0353-4

31. Powell JW, Barber-Foss KD. Sex-related injury patterns among selected high school sports. Am J Sports Med. (2000) 28:38591. doi: 10.1177/03635465000280031801

32. Pasulka J, Jayanthi N, McCann A, Dugas LR, LaBella C. Specialization patterns across various youth sports and relationship to injury risk. Phys Sportsmed. (2017) 45:344-52. doi: 10.1080/00913847.2017. 1313077

33. DiSanti JS, Erickson K. Youth sport specialization: a multidisciplinary scoping systematic review. J Sports Sci. (2019) 37:2094105. doi: 10.1080/02640414.2019.1621476

34. Hall R, Foss KB, Hewett TE, Myer GD. Sport specialization's association with an increased risk of developing anterior knee pain in adolescent female athletes. J Sport Rehabil. (2015) 24:31-5. doi: 10.1123/jsr.2013-0101

35. Barnes JD, Cameron C, Carson V, Chaput JP, Colley RC, Faulkner GEJ, et al. Results from Canada's 2018 report card on physical activity for children and youth. J Phys Act Health. (2018) 15(Suppl. 2):S32830. doi: 10.1123/jpah.2018-0454

36. Helsen WF, Starkes JL, Van Winckel J. The influence of relative age on success and dropout in male soccer players. Am J Human Biol. (1998) 10:791-8. doi: 10.1002/(SICI)1520-6300 (1998) 10:6<791::AID-AJHB10>3.0.CO;2-1

37. Weiss MR, Petlichkoff LM. Children's motivation for participation in and withdrawal from sport: identifying the missing links. Pediatric Exerc Sci. (1989) 1:195-211. doi: 10.1123/pes.1.3.195

38. Borschneck D. The Association Between Hamstring Contracture and Anterior Knee Pain in Children Aged 10-15 Years. Ottawa: National Library of Canada: Queen's University (2004).

Conflict of Interest: The authors declare that the research was conducted in the absence of any commercial or financial relationships that could be construed as a potential conflict of interest.

Publisher's Note: All claims expressed in this article are solely those of the authors and do not necessarily represent those of their affiliated organizations, or those of the publisher, the editors and the reviewers. Any product that may be evaluated in this article, or claim that may be made by its manufacturer, is not guaranteed or endorsed by the publisher.

Copyright (c) 2021 Borschneck, St. John, Brundage and Borschneck. This is an openaccess article distributed under the terms of the Creative Commons Attribution License (CC BY). The use, distribution or reproduction in other forums is permitted, provided the original author(s) and the copyright owner(s) are credited and that the original publication in this journal is cited, in accordance with accepted academic practice. No use, distribution or reproduction is permitted which does not comply with these terms. 